\title{
ELECTRIC WRIST ROTATION IN PROPORTIONAL-CONTROLLED SYSTEMS
}

\author{
Harold H. Sears, Ph.D. \\ General Manager, Motion Control Division of IOMED \\ Salt Lake City, UT \\ and \\ Julie Shaperman, O.T.R. \\ Rehabilitation Engineering Center \\ Rancho Los Amigos Medical Center \\ Downey, CA
}

\begin{abstract}
Electric wrist rotation has been available with electric hands for approximately 20 years. Although it is integrated with the Otto Bock electric hand, via their quick-disconnected wrist component, the electric wrist has been used in only a fraction of electric prostheses because of four perceived problems:

1) the additional length required in he forearm, which could eliminate mid-to-long transradial amputees as candidates,

2) the additional weight of the component, although only about $100 \mathrm{gm}$.

3) the speculation of most prescribing MD's and prosthetists that the function of electric writs rotation is unnecessary and unused by amputees, and

4) the difficulty of using the traditional wrist control methods.
\end{abstract}

The introduction in 1989 of a proportional controller, which allows a simple switching method using muscles cocontraction, possibly makes an impact on problems \#3 and \#4 above. The control method is speculated to be easier to learn and to use, and also allows proportional control of the wrist rotation. Powered by a 9 volt battery, or 12 volt in above-elbow applications, greater speed of wrists rotation is also provided.

The purpose of this survey is to evaluate the actual usage of electric wrist by the wearers of proportional systems, and to document the functions in which electric wrist rotation has proved valuable, if any. Also, the survey hopes to provide indications of the present drawbacks which remain, and provide direction for future improvements.

The results to-date of the survey will be presented, and any conclusions which are warranted by the data. Future developments which could impact upon electric prosthesis usage will also be discussed. 